\title{
PRODUCTION OF A WHITE BEAN LACKING THE FACTOR FOR TOTAL PIGMENTATION-A PROPHECY FULFILIED.
}

\author{
R. A. EMERSON, Lincoln, Neb.
}

Some years ago I called attention to the fact that certain crosses of eyed (partially pigmented) beans with white (non-pigmented) ones resulted in totally pigmented $F_{1}$ individuals. ${ }^{\circ}$ In a more recent paper ${ }^{\circ}$ I showed that the factor for total pigmentation must be latent in the white parent and predicted that the white beans occurring in $F_{2}$ would be found to consist of two types one with and the other without latent total pigmentation. At that time I knew three races of white beans that had the total-pigmentation factor and none that lacked it. During the past year I have tested several white races for latent total pigmentation by crossing them with partially pigmented races. The tests to date have included the following white races, fifteen in all: Burpee White, Crystal, Davis, Jones Stringless, Marrow, Marrow Pea, Michigan, Medium, Navy, Oliver, Sickle, Snowflake, Tree, Triumph, and Vineless Marrow. Nine races of eyed beans have been used in these tests, namely: China, Detroit, Golden Wax, Grenell, Hybrid 57, Keeney, Roundpod Kidney, Wardwell, and Yellow Eye. In all these cases, including 92 individuals, the. $F_{1}$ seeds were totally pigmented, showing that all the white races tested have the factor for total pigmentation. It 'is probable that white races exist that lack this factor but I have not as yet found any such.

My attempts to produce a white bean without the total-pigmentation factor have been more successful. A cross of the white race Navy. with the eyed race Wardwell resulted in totally pigmented seeds in $F_{1}$ and in totally pigmented, eyed, and white seeds in $F_{2}$ as was expected. None of the $F_{2}$ whites were tested but some of them were grown another generation, the $\mathrm{F}_{3}$ plants all breeding true of course to the character of white seeds. Several of these $F_{3}$ whites were crossed with one or more of the following eyed races, namely: China, Golden Eye, Grenell, and Roundpod Kidney. Most of these crosses gave totally pigmented $F_{1}$ seeds, but a few yielded eyed beans in $F_{1}$, a result not secured before in any cross. Considering the impossibility of foretelling which one of the white-seeded parent plants would give. one result and which one the other, I was extremely fortunate in

a Ann. Rpt. Nebr. Agr. Expt. Sta., 15:41 (1902).

bibid., 22 :73-80 (1909). 
securing just the combination of crosses best calculated to demonstrate the existence of the two classes of white beans. White bean family No. 56, individual No. 1, was cross-pollinated by the eyed race Grenell, individual No. 3 , and yielded in $F_{1}$ totally pigmented seeds. Another white bean, family No. 55, plant No. 2 , was crossed by the same eyed race, Grenell, the pollen having been taken from the same individual, No. 3. The result was partially pigmented (eyed) beans. in $F_{1}$. As though this were not enough, I find that the same two white-seeded plants, $56_{1}$ and $55_{2}$, were also crossed by the eyed race Roundporl Kidney, the pollen for both crosses in this case also having been taken from a single individual plant, No. 10. And in $F_{1}$ from these crosses, just as with Grenell pollen, white seeded plant $56_{1}$ yielded totally pigmented seeds and plant $55_{2}$ partially pigmented ones.

\title{
APPLICATION OF THE PRINCIPLES OF HEREDITY TO THE IMPROVEMENT OF PLANTS AND ANIMALS.
}

\author{
W. J. Spillman, Washington, D. C.
}

With reference to the principles involved in their improvement by selection and breeding, we may divide animals and plants into three general classes. It happens that the higher animals are all found in the third of these classes while plants are divided among the three classes.

The first class includes the crops that are not ordinarily propagated from seed, such as apples, peaches, pears, Irish potatoes, sweet potatoes and those varieties of onions which are propagated from sets.

The second class includes those crops that are propagated from seeds but in which, under ordinary conditions, there is little or no cross fertilization. The most important crops in this class are wheat, oats, and barley.

The third class includes those organisms in which cross fertilization normally occurs. It includes all the higher animals. Amongst plants there are some species in which cross pollination is necessary to seed production, but generally speaking most plants in this class are what we describe as open pollinated. That is, they may be pollinated in part by their own pollen and in part by pollen from neighboring plants of the same general character. Corn, timothy, clover, alfalfa and, to some extent, rye belong in this class. 